\title{
On Determinantal Symmetric Functions
}

By ZIA-ED-DrN (UTniversity of Edinburgh).

(Received 30th January, 1934. Read 2nd February, 1934).

$\S 1$. The theory of symmetric polynomials abounds in dual identities and symmetries of various kinds. It has been investigated from the determinantal standpoint largely by means of quotients of alternants, such as,

$$
\left|a^{a} b^{\beta} c^{\gamma} \ldots\right| \div\left|a^{0} b^{1} c^{2} \ldots\right|
$$

the denominator being the difference product of $a, b, c, \ldots$, a simple alternant.

This quotient, which has been called by Muir a bi-alternant, was first considered in detail by Jacobi, who gave in 1841, its equivalent as

$$
\left|\begin{array}{llll}
h_{a} & h_{\beta} & h_{\gamma} & \ldots \\
h_{a-1} & h_{\beta-1} & h_{\gamma-1} & \ldots \\
h_{a-2} & h_{\beta-2} & h_{\gamma-2} & \ldots \\
\ldots \ldots & \ldots & \ldots & \ldots
\end{array}\right|
$$

the elements $h_{r}$ being complete homogeneous or "Aleph" symmetric functions in $a, b, c, \ldots$, of degree $r$. We shall denote this type of isobaric determinant by

$$
h\left(\begin{array}{llll}
a & \beta & \gamma & \cdots \\
0 & 1 & 2 & \ldots
\end{array}\right)
$$

the upper indices being those of the elements in the first row of the bi-alternant, the lower indices being the index-differences between the first row and the various rows in order.

Naegelsbach in 1871, gave an equivalent form in elementary symmetric functions $C_{r}$, namely in our notation

$$
C\left(\begin{array}{cccc}
a & \beta & \gamma & \cdots \\
0 & 1 & 2 & \cdots
\end{array}\right)^{\prime}
$$

where it is meant by ()$^{\prime}$ that the two rows of indices in the bracket are dual or bicomplementary to $(\alpha \beta \gamma \ldots)$ and $\left(\begin{array}{lll}0 & 1 & 2\end{array} \ldots\right)$ respectively. Dual sets are defined thus:

To obtain the dual of $(a \beta \gamma \ldots \nu)$ with respect to $\left(\begin{array}{lll}0 & 1 & 2\end{array} \ldots \nu\right)$ we write in reversed order the members missing in $(\alpha \beta \gamma \ldots \nu)$ and 
then replace each one by its complement with respect to $v$, that is any $\delta$ by $\nu-\delta$, and so on. For example the dual of (0134) with respect to (012345) is (03). The dual of a set in natural order $(0123 \ldots)$ is another set in natural order.

MacMahon in combinatory investigations found dualities in isobaric determinants of a rather different type. His theorem, though expressed by him in terms of "Compositions of integers," can be expressed as follows,

$$
h\left(\begin{array}{lllllr}
\alpha & \beta & \gamma & \ldots & \mu & \nu \\
0 & \alpha & \beta & \ldots & \ldots & \mu
\end{array}\right)=C\left(\begin{array}{llllll}
\alpha & \beta & \gamma & \ldots & \mu & \nu \\
0 & \alpha & \beta & \ldots & \ldots & \mu
\end{array}\right)^{\prime},
$$

the lower indices indicating as before index-difference of rows from first row, and the pairs of sets being dual as before.

Bi-alternants, their minors and the above determinant of MacMahon are all special cases of the general isobaric determinants,

$$
h\left(\begin{array}{llll}
\alpha & \beta & \gamma & \ldots \\
0 & p & q & \ldots
\end{array}\right)
$$

a type which has been considered by Naegelsbach, Segar, Muir, Nanson, Roe, and Aitken, and which is the subject of an important general theorem by Roe. ${ }^{1}$

Aitken has given the theorem of duality which can be expressed as follows,

$$
h\left(\begin{array}{llll}
a & \beta & \gamma & \ldots \\
0 & p & q & \ldots
\end{array}\right)=C\left(\begin{array}{llll}
a & \beta & \gamma & \ldots \\
0 & p & q & \ldots
\end{array}\right)^{\prime},
$$

which includes the Jacobi-Naegelsbach and MacMahon cases.

Our object now is to express such a general isobaric determinant as a linear function of the bi-alternants, and more generally to show the relationship between identities in isobaric determinants and identities in alternants and monomial symmetric functions.

$\S 2$. Since the theory of symmetric functions depends largely on the rules for multiplying alternants by monomial symmetric functions, we may take the opportunity to express these rules concisely.

We denote an alternant $\left|a^{a} b^{\beta} c^{\gamma} \ldots\right|$ by $A(\alpha \beta \gamma \ldots)$ or by $(\dot{a} \dot{\beta} \dot{\gamma} \ldots)$ the dots indicating permutation, with determinantal sign, of the indices affected, and summation of all terms so obtained. The

1 Muir, History of Determinants, 1900-1920 (Glasgow, 1930), 186. 
monomial symmetric function $\Sigma a^{\alpha} b^{\beta} c^{\gamma} \ldots$ we denote by $P(a \beta \gamma \ldots$ ) or by $\left(\begin{array}{l}\dot{\alpha} \\ \beta\end{array}\right.$ of terms, all taken positively. Thus the relation of monomial symmetric function to alternant is that of permanent to determinant.

\section{RuLEs.}

(1) $P\left(a_{1} \beta_{1} \gamma_{1} \ldots\right) P\left(a_{2} \beta_{2} \gamma_{2} \ldots\right)=P\left(a_{1}+\stackrel{+}{a_{2}}, \beta_{1}+\stackrel{+}{\beta_{2}}, \gamma_{1}+\stackrel{+}{\gamma_{2}}, \ldots\right)$

(2) $A\left(a_{1} \beta_{1} \gamma_{1} \ldots\right) P\left(a_{2} \beta_{2} \gamma_{2} \ldots\right)=A\left(\alpha_{1}+\stackrel{+}{a_{2}}, \beta_{1}+\stackrel{+}{\beta_{2}}, \gamma_{1}+\stackrel{+}{\gamma_{2}}, \ldots\right)$

(3) $A\left(a_{1} \beta_{1} \gamma_{1} \ldots\right) A\left(\alpha_{2} \beta_{2} \gamma_{2} \ldots\right)=P\left(\alpha_{1}+\dot{\alpha}_{2}, \beta_{1}+\dot{\beta}_{2}, \gamma_{1}+\dot{\gamma}_{2}, \ldots\right)$.

The proof of rule (2) is given by Muir and Metzler ${ }^{1}$; other rules can similarly be proved.

The right members will give all the required terms without omission and with correct sign.

\section{§3. Theorem.}

If the bi-alternant $h\left(\begin{array}{cccc}0 & p & q & \ldots \\ 0 & 1 & 2 & \ldots\end{array}\right)$ be expanded in terms of monomial symmetric functions as

$$
h\left(\begin{array}{llll}
0 & p & q & \ldots \\
0 & 1 & 2 & \ldots
\end{array}\right)=\ldots+\lambda_{i j k . .} P(i j k \ldots)+\ldots
$$

the expansion being indicated by a single typical term, then

$$
h\left(\begin{array}{llll}
a & \beta & \gamma & \ldots \\
0 & p & q & \ldots
\end{array}\right)=\ldots+\lambda_{i j k . .} \sum_{i j k . .} h\left(\begin{array}{cccc}
a-i & \beta-j & \gamma-k & \ldots \\
0 & 1 & 2 & \ldots
\end{array}\right)
$$

where $\Sigma$ indicates permutation of $i, j, k \ldots$, and summation.

Proof.

Let $A$ be an operator affecting elements in the first column only (that is those with suffixes involving a) such that $A_{a-i}^{h}=_{a-i-1}^{h}$, while $B, C, \ldots$ are similar operators affecting the later columns. These operators act independently of each other and obey the fundamental laws of Algebra.

Thus we have

$$
h\left(\begin{array}{cccc}
a & \beta & \gamma & \ldots \\
0 & p & q & \ldots
\end{array}\right)=\left|A^{0} B^{p} C^{q} \ldots\right| h_{a} h_{\beta} h_{\gamma} \ldots \ldots,
$$

as can be seen at once by expanding the symbolic alternant operator.

1 Muir and Metzler, “Theory of Determinants" (1930), p. 344. 
But we have also by Jacobi's theorem on bi-alternants,

$$
\left|A^{0} B^{p} C^{q} \ldots\right|=\ldots+\lambda_{i j k} \ldots||^{i} B^{j} C^{k} \ldots+|| A^{0} B^{1} C^{2} \ldots \mid+\ldots
$$

and so operating with both sides on $h_{a} h_{\beta}^{\prime} h_{\gamma} \ldots$ we obtain

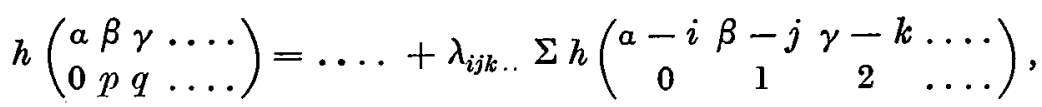

which was required to be proved.

Example. It being known from tables of symmetric functions or otherwise that

we have

$$
h\left(\begin{array}{lll}
0 & 2 & 4 \\
0 & 1 & 2
\end{array}\right)=\Sigma a^{2} b+2 a b c
$$

$$
\begin{aligned}
& h\left(\begin{array}{lll}
\alpha & \beta & \gamma \\
0 & 2 & 4
\end{array}\right)=h\left(\begin{array}{crr}
\alpha-2 \beta-1 & \gamma \\
0 & 1 & 2
\end{array}\right)+h\left(\begin{array}{ccc}
\alpha-2 & \gamma-1 \\
0 & 1 & 2
\end{array}\right)+\ldots \\
& +2 h\left(\begin{array}{ccc}
a-1 & \beta-1 & \gamma-1 \\
0 & 1 & 2
\end{array}\right)
\end{aligned}
$$

which can be easily verified on expansion.

Thus general isobaric determinants can be investigated by means of bi-alternants and the known expansions of bi-alternants.

The theorem given above is merely one case of the general result that an identity bilinear in alternants and monomial symmetric functions yields a corresponding identity in isobaric determinants. To take a simple example, by the multiplication theorem $\$ 2(2)$, we may find

$$
A(024)=A(013) P(011)-A(012) P(111) .
$$

Writing this in operational form and taking $h_{\alpha} h_{\beta} h_{\mathrm{y}}$ as operand we get $h\left(\begin{array}{lll}\alpha & \beta & \gamma \\ 0 & 2 & 4\end{array}\right)=h\left(\begin{array}{ccc}a & \beta-1 & \gamma-1 \\ 0 & 1 & 3\end{array}\right)+h\left(\begin{array}{ccc}\alpha-1 & \beta & \gamma-1 \\ 0 & 1 & 3\end{array}\right)$

which can be verified.

$$
+h\left(\begin{array}{ccc}
\alpha-1 & \beta-1 & \gamma \\
0 & 1 & 3
\end{array}\right)-h\left(\begin{array}{ccc}
\alpha-1 & \beta-1 & \gamma-1 \\
0 & 1 & 2
\end{array}\right) \text {, }
$$

\$. In the proof of these results the fact that $h_{\tau}$ in the operand is a symmetric function has not been used. In fact these identities belong to a type characteristic of independent functional operations generally. For example, the purely determinantal analogue of the first example of $\S(3)$ is

$$
\left|\begin{array}{lll}
a_{0} & b_{0} & c_{0} \\
a_{2} & b_{2} & c_{2} \\
a_{4} & b_{4} & c_{4}
\end{array}\right|=\left|\begin{array}{lll}
a_{2} & b_{1} & c_{0} \\
a_{3} & b_{2} & c_{1} \\
a_{4} & b_{3} & c_{2}
\end{array}\right|+\ldots+2\left|\begin{array}{lll}
a_{1} & b_{1} & c_{1} \\
a_{2} & b_{2} & c_{2} \\
a_{3} & b_{3} & c_{3}
\end{array}\right|
$$


or again, for a minor of a Wronskian in terms of Wronskians,

$$
\begin{array}{lll}
f & g & h \\
f^{\prime \prime} & g^{\prime \prime} & h^{\prime \prime} \\
f^{\prime \mathrm{v}} & g^{\prime \mathrm{v}} & h^{\prime \mathrm{r}}
\end{array}\left|=\sum_{f, g, h}\right| \begin{array}{lll}
f^{\prime \prime} & g^{\prime \prime \prime} & h \\
f^{\prime \prime \prime} & g^{\prime \prime} & h^{\prime} \\
f^{\prime \prime \prime} & h^{\prime \prime}
\end{array}|+2| \begin{array}{lll}
f^{\prime} & g^{\prime} & h^{\prime} \\
f^{\prime \prime} & g^{\prime \prime} & h^{\prime \prime} \\
f^{\prime \prime \prime} & g^{\prime \prime \prime} & h^{\prime \prime \prime}
\end{array} \mid .
$$

Many other examples might be given of expansions of this kind.

$\S(5)$. By Aitken's theorem of duality we have

$$
h\left(\begin{array}{llll}
a & \beta & \gamma & \ldots \\
0 & p & q & \ldots
\end{array}\right)=C\left(\begin{array}{llll}
a & \beta & \gamma & \ldots \\
0 & p & q & \ldots
\end{array}\right)^{\prime} .
$$

Each side can be expanded linearly in bi-alternants, the terms corresponding to those in the expansions of

$$
h\left(\begin{array}{rrrr}
a & \beta & \gamma & \ldots \\
0 & p & q & \ldots
\end{array}\right) \text { and } h\left(\begin{array}{llll}
0 & p & q & \ldots \\
0 & 1 & 2 & \ldots
\end{array}\right)^{\prime} \text {, that is } C\left(\begin{array}{llll}
0 & p & q & \ldots \\
0 & 1 & 2 & \ldots
\end{array}\right),
$$

in monomials. The variables being arbitrary, each $h$-bi-alternant term in the expansion must be in Jacobi-Naegelsbach duality with a $C$ bi-alternant in the other. We thus have a very peculiar theorem as follows:

TheOREM.

If any arbitrary alternant $A(\alpha \beta \gamma \ldots)$ has its indices diminished by those occuring in the terms in the expansion of $h\left(\begin{array}{llll}0 & p & q & \ldots \\ 0 & 1 & 2 & \ldots\end{array}\right)$ in monomials, and if in the aggregate of alternants so obtained each alternant is replaced by its dual, the resultiug set is identical with that obtained by diminishing the indices of the dual alternant $A(\alpha \beta \gamma \ldots)^{\prime}$ by those of the terms of the expansion of $C\left(\begin{array}{llll}0 & p & q & \ldots \\ 0 & 1 & 2 & \ldots\end{array}\right)$ in monomials.

To be precise we must take the duality to be with respect to such a set $(012 \ldots \nu)$ that the orders of the alternants are equal. (This is necessary in order that the number of variables in the dual alternants may be the same.)

Example. $\quad h_{2}=\Sigma a^{2}+\Sigma a b$ and $C_{2}=\Sigma a b$ are simple examples of dual bi-alternants and $A(1456)$ and $A(0457)$ are dual alternants. Operating on $A(1456)$ with an operator corresponding to $h_{2}$ we derive

$$
A(1256)+A(1436)+A(0356)+A(1346)=A(1256)+A(0356),
$$


and operating on $A(0457)$ with an operator corresponding to $C_{2}$ we get

$$
A(0347)+A(0356),
$$

other terms vanishing. This is in agreement with the theorem, for the dual of (1256) is (0347), while (0356) is self dual.

A direct proof of this peculiar property of dual sets appears to be difficult.

\section{REFERENCES.}

1. Historical details of the progress of bi-alternant theory will be found in Muir's "History of Determinants," Vols. 1-4, and the "History of Determinants," 1900-1920.

2. Aitken, Proc. Edin. Math. Soc. (2), 1 (1927), 55-61 ; 2 (1931), 164.7.

.3. MacMahon, "Combinatory Analysis," 1 (1915), 205. 